\title{
PROCESSO DE ASSISTÊNCIA AO PARTO NORMAL EM UMA MATERNIDADE PÚBLICA DO ESTADO DO PIAUÍ, 2015.
}

\section{ASSISTANCE PROCESS TO NORMAL BIRTH IN A PUBLIC MATERNITY OF PIAUI, 2015.}

\section{PROCESO DE SOPORTE DE NACIMIENTO NORMAL EN UNA MATERNIDAD PÚBLICA DE PIAUI, 2015.}

\author{
Bruna Fernandes Almeida ${ }^{1}$, José Francisco Ribeiro ${ }^{2}$, Kleiton Richard da Silva Araújo ${ }^{3}$, Tássio \\ Breno de Sousa Lopes Lavôr ${ }^{4}$
}

\begin{abstract}
RESUMO
OBJETIVO: analisar o processo de assistência ao parto natural em uma maternidade pública de referência para o estado do Piauí. MÉTODOS: estudo descritivo, com abordagem quantitativa, realizado com 120 puérperas que tiveram parto normal e admitidas nas enfermarias, a coleta de dados ocorreu no período de abril e maio de 2015 , por meio de um formulário previamente elaborado pelos pesquisadores, para a análise dos dados utilizou-se estatística descritiva. RESULTADOS: dinâmica uterina 8,3\%, toque vaginal 99,2\%, ausculta dos BCF 98,3\%, controle não farmacológico da dor 32,5\%, preenchimento do partograma $68,3 \%$, presença do acompanhante $58,3 \%$, episiotomia $18,3 \%$, posição semi-vertical $90 \%$, contato pele-a-pele mãe e bebê $95,8 \%$, aleitamento materno na primeira hora de vida 77,5\%. CONCLUSÕES: o processo de assistência ao parto natural está sendo realizado de forma adequada, mas sendo necessária a busca de novos estudos que possibilitem identificação de novos procedimentos para diminuir ou sanar as dificuldades encontradas.
\end{abstract}

PALAVRAS-CHAVE: Parto normal. Salas de parto. Parto humanizado.

\begin{abstract}
OBJECTIVE: To analyze the natural childbirth care process in a public maternity in reference to the state of Piauí. METHODS: A descriptive study with a quantitative approach, conducted with 120 mothers who had normal birth and admitted in the wards, data collection took place between April and May 2015, through a form previously prepared by the researchers for the analysis of data was used descriptive statistics. RESULTS: uterine dynamics $8.3 \%$, 99.2\% vaginal examination, auscultation of $\mathrm{BCF} 98.3 \%$, nonpharmacological control of pain $32.5 \%$, partogram completing $68.3 \%, 58.3 \%$ of the partner's presence, episiotomy $18.3 \%$, semi-upright $90 \%$, skin-to-skin mother and baby $95.8 \%$, breastfeeding in the first hour of life $77.5 \%$. CONCLUSIONS: the natural childbirth care process is being carried out properly, but is necessary to search for new studies for identifying new procedures to reduce or remedy the difficulties encountered.
\end{abstract}

KEYWORDS: Natural Childbirth. Delivery Rooms. Humanizing Delivery.

\footnotetext{
RESUMEN

${ }^{1}$ Enfermeira graduada pela UESPI. E-mail: brunauespi@ gmail.com.

${ }^{2}$ Mestre em Ciências e Saúde pelo programa de Pós-graduação da Universidade Federal do Piauí (UFPI) e docente do curso de graduação em enfermagem da UESPI/FACIME. E-mail: jotafribeiro@ yahoo.com.br.

${ }^{3}$ Enfermeiro graduado pela UESPI. Pós-Graduando em Enfermagem Obstétrica (FVJ) e docente do curso de graduação em Enfermagem da Faculdade do Médio Parnaíba (FAMEP). E-mail: kleitonrich@ gmail.com.

${ }^{4}$ Enfermeiro graduado pela UESPI. E-mail: tassio.breno@ hotmail.com.
} 
OBJETIVO: Analizar el proceso de atención al parto natural en una maternidad pública en referencia al estado de Piauí. MÉTODOS: Se realizó un estudio descriptivo con un enfoque cuantitativo, realizado con 120 madres que tuvieron un parto normal y admitidas en las salas, la recogida de datos se llevó a cabo entre abril y mayo de 2015, a través de un formulario preparado previamente por los investigadores para el análisis de los datos se utilizó la estadística descriptiva. RESULTADOS: dinámica uterina 8,3\%, examen vaginal 99,2\%, auscultación del BCF 98,3\%, el control no farmacológico del dolor 32,5\%, 68,3\% partograma que terminan, el 58,3\% de la presencia de la pareja, episiotomía $18,3 \%$, semi-vertical $90 \%$, la madre de piel a piel y el bebé $95,8 \%$, la lactancia materna en la primera hora de vida del 77,5\%. CONCLUSIONES: el proceso de atención de parto natural se está llevando a cabo correctamente, pero es necesaria la búsqueda de nuevos estudios para la identificación de nuevos procedimientos para disminuir o mitigar las dificultades encontradas.

PALABRAS CLAVE: parto normal. sala de partos. Humanización del parto.

\section{INTRODUÇÃO}

As taxas de parto normal no Brasil tiveram uma diminuição, como pode ser observado em um estudo realizado no Paraná de 2002 a 2012 no qual o parto normal no Sistema Único de Saúde (SUS) diminuiu de 58,1 para 34,7\%, demonstrando que apesar das recomendações e incentivos ao parto normal SUS ainda não é uma realidade no país, sendo que há uma prevalência dos partos operatórios. ${ }^{1}$

O parto natural é um tipo de parto realizado sem intervenções desnecessárias e sem procedimentos de maior complexidade, durante o trabalho de parto, parto e pós parto, sendo realizado um atendimento centralizado na mulher. É também conhecido como parto humanizado, pois é realizado com respeito e com ternura à mulher e ao filho durante esse período. $^{2}$
Observa-se que houve uma despersonalização do processo de parir, sendo que os principais autores do parto passaram a ser os médicos e enfermeiros, ao invés da mulher com o filho. O hospital passa a ser colocado como um ambiente controlado e seguro e o profissional de saúde é quem conduz o processo de parto. ${ }^{3}$

Muitas mulheres apresentam insegurança no que concerne ao parto e ao bem-estar do feto e confiam plenamente no profissional de saúde que as assistem. A esses profissionais compete à responsabilidade sobre as orientações adequadas de acordo com as evidências científicas. ${ }^{4}$

Existem diversos benefícios em um parto natural tanto para a mulher quanto para o bebê. Diante disso pode-se citar o alívio da dor durante o trabalho de parto e parto que é favorecido com a realização de massagens, banhos de chuveiro e em banheiras de hidromassagem, musicas e 
outras técnicas de relaxamento, manobras que fazem com que a mulher sinta menos dor, pois o foco da mulher não será mais a dor, podendo participar de maneira mais ativa e autônoma no processo de parto. A parturiente conta com a presença de um acompanhante escolhido por ela, é um direito garantido por lei, o uso de técnicas não farmacológicas e a presença do acompanhante, diminuem a dor relacionada ao trabalho de parto. ${ }^{2}$

Levando-se em conta a humanização durante o processo de parto, torna-se necessário respeitar o tempo que a mulher requer durante o processo de parir, evitar intervenções desnecessárias que são realizadas devido a rotinas hospitalares e reconhecer e respeitar os aspectos culturais da mesma. Nesse tipo de parto existe diversas vantagens como uma recuperação mais rápida, menor risco de infecção e maior satisfação da parturiente. Quanto ao aleitamento materno na primeira hora de vida do recém-nascido, em uma pesquisa realizada pelo Ministério da Saúde, mostra que $22,4 \%$ das mulheres logo após o parto normal iniciam o aleitamento materno, já das que realizam cesariana apenas $5,8 \%$ iniciam essa prática., ${ }^{5,6}$

Dentre as melhorias alcançadas, pode-se citar a Rede Cegonha, que foi lançada em 2011, no Brasil, como uma estratégia inovadora que consiste em uma rede de cuidados que visa assegurar à mulher o direito ao planejamento reprodutivo, uma atenção humanizada durante a gravidez, o parto, o puerpério e o abortamento, e à criança o direito ao nascimento seguro e crescimento e desenvolvimento saudáveis. ${ }^{7,8}$

O parto normal vem sendo cada vez mais estimulado, em decorrência do grande número de partos cesarianos que vem sendo feitos no Brasil, o presente estudo é uma forma de saber se esse tipo de parto está sendo realizado de maneira adequada na maternidade onde se desenvolveu a pesquisa, podendo proporcionar melhorias na instituição de saúde por meio dos resultados encontrados, que se configura em benefícios para as usuárias do serviço. Objetivou analisar o processo de assistência ao parto natural em uma maternidade pública de referência para o estado do Piauí, no ano de 2015.

\section{MÉTODOS}

Estudo de natureza exploratório descritivo, com abordagem quantitativa, realizado por meio de entrevista com puérperas que tiveram parto normal na instituição em que ocorreu a pesquisa, e por meio de informações obtidas no prontuário da mulher e do recém-nascido. 
$\mathrm{O}$ estudo foi desenvolvido em Teresina, capital do Estado do Piauí, que tem aproximadamente uma população de 822.363 habitantes, localizada no centro norte piauiense (IBGE, 2010). ${ }^{9}$

As participantes deste estudo foram puérperas de parto normal admitidas nas enfermarias provenientes do Centro de Parto Normal (CPN) de uma maternidade de referência no estado do Piauí. Aproximadamente 170 parturientes são admitidas mensalmente no CPN. Para esse estudo a amostra foi composta por 120 puérperas considerando-se o índice de confiabilidade (IC) de $95 \%$, erro de $5 \%$. Apresentando como critérios de inclusão as puérperas que realizaram parto normal na maternidade e que aceitaram participar do estudo, e como critérios de exclusão mulheres que tiveram parto normal fora das dependências do CPN, ou com alguma intercorrência clínica no momento da coleta de dados.

A coleta de dados ocorreu entre Abril e Maio de 2015, nas enfermarias, local destinado ao tratamento pós parto imediato e mediato, por meio de um instrumento previamente elaborado pelos pesquisadores, diretamente com as puérperas e por meio do prontuário da mesma e do recém-nascido (RN), e mediante a assinatura do Termo de Consentimento Livre e Esclarecido
(TCLE), pelo Termo de Assentimento em caso de menores de idade, e pelo Termo de Fiel Depositário.

No instrumento de coleta de dados eram abordados pontos como: estrutura do CPN, procedimentos e classificação da adequação dos procedimentos clínicos obstétricos realizados no $\mathrm{CPN}$, caracterização sociodemografica das puerperas que realizaram parto no $\mathrm{CPN}$. Após a coleta os dados foram analisados por meio do programa estatístico Statistical Package for Social Science. (SPSS) versão 20.0, no qual os dados foram resumidos por meio de tabelas.

A pesquisa respeitou os preceitos éticos da Resolução 466/2012 do Conselho Nacional de Saúde que trata das diretrizes e normas regulamentadoras de pesquisa envolvendo seres humanos. Foi registrada na Plataforma Brasil do Sistema Nacional de Ética e Pesquisa (SISNEP) e posteriormente, aprovada pelo Comitê de Ética do Hospital São Marcos CEP/HSM de acordo com o Certificado de Apresentação para Apreciação Ética CAAE número 39470414.0.0000.5584, foi autorizada pela instituição coparticipante através do Termo de Utilização de Dados (TUD) e da Carta de Anuência. 


\section{RESULTADOS}

No serviço de admissão verificouse nas parturientes a dinâmica uterina em $98,3 \%$; o toque vaginal em 99,2\%, a pressão arterial (PA) em 96,7\%, foi realizado à ausculta de batimentos cardíacos fetais (BCF) em 98,3\%, não houve a realização de enteroclisma e tricotomia.

Quanto aos procedimentos e rotinas da sala de pré-parto 18 mulheres não foram avaliadas nesse ambiente, pois foram assistidas diretamente na sala de parto, por darem entrada na maternidade já em período expulsivo. Foi predominante no pré-parto a ingestão de líquidos doces e água $79,2 \%$, a deambulação 81,7\%, o controle não farmacológico da dor $32,5 \%$, a ausculta dos BCF 84,2\%, quanto ao preenchimento do partograma 68,3\% dos prontuários apresentaram esse em branco e o acompanhante se fez presente em $74,1 \%$ dos partos.

$\mathrm{Na}$ sala de parto a presença do acompanhante foi de $58,3 \%$, não foi realizada a episiotomia em $81,7 \%$ das parturientes, a administração da ocitocina no terceiro estágio do parto ocorreu na totalidade das parturientes, o contato pelea-pele da mãe com o $\mathrm{RN}$ foi $95,8 \%$, a condução do parto por enfermeiro obstetra foi de $21,7 \%$ dos partos, a manobra de Kristeller não foi evidenciada em $80 \%$ dos partos, e a maioria das mulheres adotaram a posição semi-vertical $90 \%$,

$\mathrm{Na}$ assistência ao neonato, houve atendimento por pediatra ou graduando de medicina na sala de parto $100 \%$, administração de vitamina $K$, índice de apgar, idade gestacional por exame físico, identificação do peso, estatura e perímetro cefálico $100 \%$, coleta de sangue para a tipagem sanguínea $60,8 \%$, para VDRL $60 \%$, para exame anti-HIV $0,8 \%$, e o aleitamento materno na primeira hora de vida foi relatado por $77,5 \%$ das puérperas.

Tabela 01- Procedimentos realizados com a parturiente na admissão, pré-parto, sala de parto e cuidados ao neonato em uma maternidade estadual. Teresina (PI), 2015. 


\begin{tabular}{|c|c|c|c|c|}
\hline \multirow{2}{*}{\multicolumn{5}{|c|}{ ADMISSÃO }} \\
\hline & & & & \\
\hline Dinâmica uterina & 118 & 98,3 & 2 & 1,7 \\
\hline Toque vaginal & 119 & 99,2 & 1 & 0,8 \\
\hline Pressão arterial & 116 & 96,7 & 4 & $\mathbf{3 , 3}$ \\
\hline Ausculta dos batimentos cardíacos fetais & 118 & 98,3 & 2 & 1,7 \\
\hline Enteroclisma eTricotomia & 0 & 0,0 & 0 & 0,0 \\
\hline \multicolumn{5}{|l|}{ SALA DE PRÉ-PARTO } \\
\hline Indicação de jejum & 7 & 5,8 & 96 & 79,2 \\
\hline Indicação de repouso & 4 & 3,8 & 98 & $\mathbf{8 1 , 7}$ \\
\hline Controle não farmacológico da dor & 39 & 32,5 & 63 & 52,5 \\
\hline Ausculta dos batimentos cardíacos fetais & 101 & 84,2 & 19 & 15,8 \\
\hline Preenchimento do partograma & 38 & 31,7 & 82 & 68,3 \\
\hline Presença de acompanhante & 89 & 74,1 & 31 & 25,9 \\
\hline \multicolumn{5}{|l|}{ SALA DE PARTO } \\
\hline Ausculta dos batimentos cardíacos fetais & 51 & 42,5 & 69 & 57,5 \\
\hline Presença do acompanhante & 70 & 58,3 & 50 & 41,7 \\
\hline Episiotomia para parto vaginal & 22 & 18,3 & 98 & $\mathbf{8 1 , 7}$ \\
\hline Ocitocina no terceiro estágio do parto & 120 & 100 & 0 & 0,0 \\
\hline Contato pele a pele mãe/bebe & 115 & 95,8 & 5 & 4,2 \\
\hline Parto conduzido por enfermeiro obstetra & 26 & 21,7 & 94 & 78,3 \\
\hline Parto conduzido por médicos, residentes ou graduandos. & 94 & 78,3 & 26 & 21,7 \\
\hline Manobras de Kristeller & 24 & 20 & 96 & 80 \\
\hline Parto na posição semi-vertical & 108 & 90 & 12 & 10 \\
\hline \multicolumn{5}{|l|}{ ASSISTENCIA AO NEONATO } \\
\hline Atendimento por pediatra ou graduando de medicina & 120 & 100 & 0 & 0,0 \\
\hline Administração de vitamina $\mathrm{K}$ & 120 & 100 & 0 & 0,0 \\
\hline Índice de Apgar no primeiro minuto de vida & 120 & 100 & 0 & 0,0 \\
\hline Idade gestacional por exame físico & 120 & 100 & 0 & 0,0 \\
\hline $\begin{array}{l}\text { Identificação do peso, estatura e } \\
\text { perímetros cefálico, torácico e abdominal }\end{array}$ & 120 & 100 & 0 & 0,0 \\
\hline Coleta de sangue para tipagem sanguínea & 73 & 60,8 & 47 & 39,2 \\
\hline Coleta de sangue para exame de VDRL & 72 & 60 & 48 & 40 \\
\hline Coleta de sangue para exame anti-HIV & 1 & 0,8 & 119 & 99,2 \\
\hline Amamentação na primeira hora de vida & 93 & 77,5 & 27 & 22,5 \\
\hline \multicolumn{5}{|l|}{ Adaptado: Parada, et al, 2007.} \\
\hline Fonte: Maternidade Pública de Teresina PI & \multicolumn{4}{|c|}{$\begin{array}{l}\text { toques deve ser o mínimo possível, haja } \\
\text { vista que toques frequentes e sem a técnica }\end{array}$} \\
\hline DISCUSSÃO & $\begin{array}{l}\text { correta são traı } \\
\text { maternos e podem }\end{array}$ & áticos & & $\begin{array}{l}\text { tecidos } \\
\text { s da }\end{array}$ \\
\hline
\end{tabular}

Um estudo realizado em 13 hospitais de Goiânia mostrou que, a avaliação da Dinâmica Uterina foi extremamente baixa, contrastando com os achados desse estudo, em que essa avaliação foi realizada em de $98,3 \%$, das entrevistadas. ${ }^{10}$

Quanto ao toque vaginal observoucérvice e propiciar infecção da genitália. $^{11,12}$

A verificação da PA é importante para saber do bem-estar materno, sedo que um aumento súbito da PA pode identificar problemas em tempo oportuno para uma boa resolução. No presente estudo se que houve essa prática em $99,2 \%$, no 
verificou-se que a PA foi avaliada em $96,7 \%$ das mulheres. ${ }^{13}$

Em protocolos de enfermagem obstétrica a ausculta dos BCF é recomendada desde o primeiro contato com a mulher e em avaliações subsequentes, pois alterações na frequência cardíaca fetal podem ser indícios de sofrimento fetal. Pesquisa realizada no CPN do hospital geral de Itapecerica da Serra mostrou que a ausculta dos BCF, foi realizada em média a cada 56 minutos durante o trabalho de parto. Nesse estudo observou-se que a ausculta dos BCF foi satisfatória na admissão com 98,3\% e na sala de pré-parto com $84,2 \% .^{14,15}$

Um estudo realizado no Sul do Brasil evidenciou, por meio de relato de trabalhadores de saúde, que aproximadamente $70 \%$ das mulheres admitidas realizaram tricotomia, realidade não existente no presente estudo. Quanto ao enteroclisma os dados corroboram com os achados desse estudo, sendo uma prática em desuso na unidade. ${ }^{16}$

Pesquisa desenvolvida em um hospital universitário no sul do Brasil mostra que a restrição hídrica e alimentar durante a parturição é comum na instituição, porém sabe-se que durante o trabalho de parto a mulher gasta muita energia, e essa restrição pode levar a um maior desconforto, além de desencadear uma hipoglicemia que favorece riscos para o feto. É uma realidade que contrasta com os achados dessa pesquisa na qual $79,2 \%$ das parturientes não foram orientadas ao jejum, podendo ingerir líquidos doces e água, e as que foram orientadas a não se alimentar foi devido à possibilidade de um parto cirúrgico. ${ }^{17}$

Em uma maternidade pública do Rio de Janeiro a deambulação está entre os cuidados relacionados ao conforto e relaxamento das mulheres, sendo que a maioria das parturientes que tiveram práticas de relaxamento como a deambulação evoluiu para o parto normal, no presente estudo foi observado que a maioria das parturientes, $81,7 \%$ referiu ter sido estimulada à deambulação. ${ }^{18}$

No que se refere ao controle não farmacológico para o alívio da dor, observou-se que $32,5 \%$ das mulheres entrevistadas referiram ter realizado banho de chuveiro, massagem nas costas, uso do cavalinho e bola de boubat. Em uma maternidade pública de Santa Catarina a massagem na região sacra e dorsal, banhos térmicos, ambiente confortável, exercícios na bola e hidratação como manobras realizadas para alívio da dor, são práticas comuns e estimuladas. Um CPN de uma maternidade localizada na cidade de Londres evidencia a importância no uso dos métodos não farmacológicos para o 
alívio da dor durante o trabalho de parto. $^{19,20}$

Em uma maternidade pública do Rio de Janeiro foi constatado que um ambiente silencioso, o conforto e relaxamento durante o trabalho de parto são cuidados que devem acontecer, sendo necessários para que os fenômenos fisiológicos da parturição se desenvolvam de forma adequada com liberação de hormônios como a ocitocina, facilitando o trabalho de parto. ${ }^{14}$

O preenchimento do partograma não foi realizado em $68,3 \%$ dos partos, porém sabe-se que na assistência obstétrica é necessário que se compreenda a importância na habilidade e uso sistemático desse instrumento, sendo necessário aos profissionais que almejam um desempenho profissional competente, humanizado e seguro durante o trabalho de parto, possibilitando a identificação de desvios da normalidade na parturição e a tomada de medidas cabíveis e em tempo oportuno visando à correção de potenciais agravos à mulher e ao feto durante o trabalho de parto. ${ }^{12}$

Quanto à presença do acompanhante $74,1 \%$ estiveram presentes na sala de pré-parto e 58,3\% na sala de parto, as que não estavam com o acompanhante foi por terem sido admitidas já em período expulsivo, por vontade do acompanhante de não entrar na sala de parto, ou por falta de roupas para entrar no centro de parto da instituição. Uma pesquisa realizada na casa de parto David Capistrano Filho, no RJ, mostra que muitas mulheres ainda enfrentam barreiras institucionais para usufruir desse direito, que é garantido pela Lei federal $11.108 / 2005$. Uma pesquisa realizada no Brasil mostrou que $75 \%$ das mulheres tiveram a presença do acompanhante em algum momento do parto. ${ }^{18,7}$

Para a maioria das mulheres não houve a realização de episiotomia, sendo que apenas 18,3\% das mulheres foram submetidas a essa prática. Em uma maternidade pública do Rio de Janeiro constatou-se que as episiotomias ocorreram em apenas 11,2\% dos partos normais, o que corrobora com os achados dessa pesquisa, sendo uma característica positiva na instituição ${ }^{17}$.

O contato pele-a-pele do RN com a mãe auxilia na regulação da temperatura do bebê, sempre que for possível deve-se manter esse contato durante a primeira hora de vida, é uma prática que não deve se limitar apenas a sala de parto, sendo importante realiza-la durante os primeiros dias de vida para regular a temperatura do $\mathrm{RN}$ e promover a amamentação, além de fortalecer o vínculo entre a mãe e o neonato. Essa é uma realidade encontrada 
na maternidade em estudo na qual em 95,8\% dos partos houve a realização dessa prática, porém como os cuidados mediatos com o bebê não ocorrem no mesmo espaço em que é realizado o parto, o neonato é retirado do colo da mãe por um curto período de tempo para a realização desses cuidados de rotina. ${ }^{15}$

Quanto ao profissional que realizou o parto obteve-se o percentual de 78,3\% feitos por médicos, residentes ou graduandos de medicina, e $21,7 \%$ por enfermeiro. Estudo mostra que a maioria dos partos realizados em uma maternidade pública foi feito por médico, $85,8 \%$ e por enfermeiro apenas $14,2 \%$, semelhante aos achados dessa pesquisa. Sendo que a enfermagem na área obstétrica vem se consolidando a cada dia, como se observa na Resolução Conselho Federal de Enfermagem (COFEN) $\mathrm{N}^{\circ}$ 478/2015, a qual normatiza a atuação e a responsabilidade civil do enfermeiro obstetra e obstetriz nas casas de parto e nos centros de parto normal; e ainda Resolução COFEN N ${ }^{\circ} 479 / 2015$ que estabelece os critérios para registro de títulos de enfermeiro obstetra e obstetriz. $^{2}$

A manobra de Kristeller foi relatada por $20 \%$ das entrevistadas, sendo que essa é uma manobra que pode levar a complicações, pois desarranja a contratilidade uterina, produz hipertonia, repercutindo de forma negativa na vitalidade fetal, podendo ainda levar a um deslocamento prematuro de placenta e embolia amniótica. ${ }^{11,18}$

A posição semi-vertical prevaleceu em $90 \%$ dos partos, os demais foram em posição sentada no banquinho, de quatro e lateral, sendo posições que foram escolhidas pela parturiente de acordo com a necessidade de conforto individual de cada uma. Estudo semelhante mostra as diversas posições adotadas em uma casa de parto dentre elas a lateral, vertical, semivertical e de cócoras. ${ }^{18}$

A antropometria é um cuidado de rotina do $\mathrm{RN}$ na sala de parto, fazendo-se a avaliação do peso, estatura, e perímetros cefálico, abdominal e torácico. A vitamina $\mathrm{K}$ deve ser administrada para prevenir sangramentos por sua deficiência. Deve-se ainda realizar a coleta de sangue da mãe e do cordão umbilical para determinação dos antígenos do sistema $\mathrm{ABO}$ e $\mathrm{Rh}$, em casos de mãe $\mathrm{Rh}$ negativo, e ainda a coleta de sangue materno para determinar sorologia para sífilis, e HIV se a mãe não tiver realizado no último trimestre da gravidez. No presente estudo observou-se que $100 \%$ dos $\mathrm{RN}$ foram atendidos por pediatra na sala de parto, tiveram suas medidas antropométricas avaliadas, vitamina $\mathrm{K}$ e boletim de Apgar; quanto a tipagem sanguínea e VDRL observou-se o resultado 
desses exames para $60,8 \%$ e $60 \%$ respectivamente, o exame de HIV foi observado em apenas $0,8 \%$ dos prontuários. $^{15}$

Quanto ao aleitamento materno na primeira hora de vida $77,5 \%$ das mulheres relataram essa prática, as mulheres que não amamentaram nesse momento relataram ter sido devido a alguma intercorrência com RN, o que impossibilitou o aleitamento materno (AM), ou por referir dificuldade do neonato em fazer uma pega adequada. O início da amamentação imediata após o nascimento assegura que o recém-nascido receba o colostro que é rico em anticorpos, antimicrobianos, antiinflamatórios e vitamina A, importantes para a proteção do bebê, além de favorecer o vínculo afetivo entre a mãe e o filho. ${ }^{13}$

\section{CONSIDERAÇÕES FINAIS}

Os esclarecimentos à luz das orientações do Ministério da Saúde evidenciaram que o processo de assistência ao parto natural está sendo realizado de forma adequada, porém, existem melhorias a serem alcançadas como é evidenciado pela baixa adesão dos profissionais ao uso do partograma, um melhor provimento de roupas para que a mulher não deixe de ter a presença do acompanhante por falta de vestimentas, e que os cuidados com o RN possam ser postergados, se tiverem uma boa adaptação à vida extrauterina, proporcionando a mulher e ao filho um momento de privacidade e respeito. $\mathrm{O}$ que nos remete a busca de novos estudos que possibilitem identificar o que pode ser feito para diminuir ou sanar as dificuldades encontradas, dentre elas o preenchimento incompleto dos dados nos prontuários que apareceu no estudo como um grande problema.

\section{REFERÊNCIAS}

1. Paris GF, Monteschio LVC, Oliveira RR, Latorre MRDO, Pelloso SM, Mathias TAF. Tendência temporal da via de parto de acordo com a fonte de financiamento. Rev Bras Ginecol Obstet. [online]. 2014; 36(12): 548-554. ISSN 0100-7203.

Disponível em: http://www.scielo.br/pdf/rbgo/v36n12/010 0-7203-rbgo-36-12-0548.pdf

2. COREN-SP. Conselho Regional de Enfermagem do Estado de São Paulo. Parto natural. São Paulo, 2010. Disponível em: http://inter.corensp.gov.br/sites/default/files/parto_natural.p df

3. Gonçalves R, Aguiar CA, Merighi MAB, Jesus MCP. Vivenciando o cuidado no contexto de uma casa de parto: o olhar das usuárias. Rev Esc Enferm USP. 2011 Mar; 45(1): 62-70. Disponível em: http://www.scielo.br/scielo.php?script=sci _arttext\&pid=S008062342011000100009\&lng=en\&nrm=iso

4. Haddad SMT, Cececatti JG. Estratégias dirigidas aos profissionais para a redução 
das cesáreas desnecessárias no Brasil. Rev Bras Ginecol Obstet. [online]. 2011; 33(5): 252-262. ISSN 0100-7203. Disponível em: http://www.scielo.br/pdf/rbgo/v33n5/a08v 33n5.pdf

5. Moreira KAP, Araújo MAM, Fernandes AFC, Braga VAB, Freitas MJ, Queiroz MVO. O significado do cuidado ao parto na voz de quem cuida: uma perspectiva à luz da humanização. Cogitare enferm. 2009 Dez; 14(4):720-728. Disponível em: http://www.revenf.bvs.br/scielo.php?script =sci_arttext\&pid=S1414-

$85362009000400017 \& \operatorname{lng}=\mathrm{es} \& \mathrm{nrm}=$ iso

6. Rocha, J. S. Parto humanizado [dissertação]. Porto Alegre: Instituto Federal de Educação, Ciência e Tecnologia do Rio Grande do Sul; 2012.

7. Brasil. Fundação Oswaldo Cruz (FIOCRUZ). Nascer no Brasil: A mãe sabe parir, e o bebê sabe como e quando nascer. Fiocruz; 2014. Disponível em: http://www.ensp.fiocruz.br/portalensp/informe/site/arquivos/anexos/nascerw eb.pdf

8. Brasil. Ministério da Saúde. Orientações para Elaboração de Propostas da Rede Cegonha [Internet]. 12 abr. 2012.

Disponível em: www.saude.pi.gov.br/ckeditor_assets/attac hments/141/MANUAL_DE_PROPOSTAS _REDE_CEGONHA.pdf

9. Santos GHN, Martins MG, Sousa MS, Batalha SC. Impacto da idade materna sobre os resultados perinatais e via de parto. Rev Bras Ginecol Obstet. [online]. 2009; 31(7): 326-334. ISSN 0100-7203.

10. Giglio, M. R. P.; França, E.; Lamounier, J. A. Avaliação da qualidade da assistência ao parto normal. Rev Bras Ginecol Obstet. 2011 Out; 33(10): 297304. Disponível em: http://www.scielo.br/pdf/rbgo/v33n10/05.p df
11. Montenegro CAB, Filho J R. Obstetrícia fundamental. 11. ed. Rio de Janeiro: Guanabara Koogan, 2011. p. 24655 .

12. Zugaib M. Obstetrícia. 2. ed. São Paulo: Malone, 2012. p. 383-94.

13. Brasil. Associação Brasileira de Obstetrizes e Enfermeiros Obstetras (ABENFO). Assistência ao parto normal: Um guia prático. Abenfo; 2009.

Disponível em:

http://abenfo.redesindical.com.br/arqs/mate ria/56_a.pdf

14. Brasil. Secretaria Municipal de Saúde do Rio de Janeiro. Protocolo assistencial de enfermagem obstétrica da secretaria municipal de saúde do Rio de Janeiro. SMS/RJ; 2013. Disponível em: http://abenfo.redesindical.com.br/arqs/man uais/161.pdf

15. Schneck CA, Riesco MLG. Intervenções no parto de mulheres atendidas em um centro de parto normal intra-hospitalar. Rev Min Enferm., Minas Gerais. 2006; 10(3): 240-246. Disponível em:

http://www.reme.org.br/artigo/detalhes/413

16. Carvalho VF, Kerber NPC, Busanello J, Costa MG, Gonçalves BG, Quadros VF. Práticas prejudiciais ao parto: relato dos trabalhadores de saúde do sul do Brasil. Ver. Rene. 2010; 11: 92-98.

17. Enderle CF, Kerber NPC, Susin LRO, Mendoza-Sassi RA. Avaliação da atenção ao parto por adolescentes em um hospital universitário. Rev. Bras. Saude Mater. Infant. 2012 dec; 12(4):383-94. Disponível em:

http://www.scielo.br/scielo.php?script=sci _arttext\&pid=S1519-38292012000400005

18. Pereira ALF, Nagipe SFSA, Lima GPV, Nascimento SD, Gouveia MSF. 
Cuidados e resultados da assistência na sala de relaxamento de uma maternidade pública, Rio de Janeiro, Brasil. Texto Contexto Enferm, 2012; 21(3):566-73. Disponível em:

http://www.scielo.br/pdf/tce/v21n3/v21n3a 11.pdf

19. Caus ECM. Santos EKA, Nassif AA, Monticelli M. O processo de parir assistido pela enfermeira obstétrica no contexto hospitalar: significados para as parturientes. Esc. Anna Nery. 2012 mar; 16(1):34-40. Disponível em: http://www.scielo.br/pdf/ean/v16n1/v16n1 a05.pdf

20. Macfarlane AJ, Rocca-Ihenacho L, Turner LR, Roth C. Survey of women's experiences of care in a new freestanding midwifery unit in an inner city area of London, England: 2. Specific aspects of care. Midwifery. 2014 may; 30:10091020. Disponível em: http://www.midwiferyjournal.com/article/S 0266-6138(14)00093-X/pdf 